\title{
Index
}

Note: Literary works are indexed separately in this index, as are programme and film titles; numbers in italics refer to illustrations; 'n.' denotes a note

2001: A Space Odyssey 72

64,000 Question, The 34

Abbott, Stacey 18

Ace Books 95-6

Ackland, Joss 37

adaptation $3,8,9,13,23-4,28-30$,

$32-4,36-55,56 n .2,56 n .8-10,58-$

$70,72-3,74,76-7,87$,

$88 \mathrm{n} .2-3,88 \mathrm{n} .11,90-2,95-121$, 121n.1-2, 121n.8, 125-6, 127-8, 150, 160n.8, 191

see also female Gothic; Gothic

literature; individual authors

Addams, Charles 127-8

Addams Family, The (1964-66) 5, 8, $16,24,122,126-31,134-5,138-$ 46,148

Addams Family, The (1973-75) 160n.3

Addams Family, The (1991) 160n.4

Addams Family, The (1992-93) 160n.3

Addams Family Reunion, The 160n.5

Addams Family Values 160n.4

Adventures of Ozzie and Harriet, The 129

Adventures of Sherlock Holmes, The $89 \mathrm{n} .11$

advertising 14-15, 16, 61, 63, 83, 161, 167,177

Akass, Kim and MacCabe, Janet 203

Alfred Hitchcock Presents 128

Alice in Wonderland 42

Allen, Douglas 30

Ally McBeal 198n.16
Altman, Rick 2

Alwyn, Jonathan 36-7

American Gothic 9-10, 24-5, 122-4, $126-7,139,140,146,156-7,159$, $161-2,165,170-1,183-8,195-7$, 200, 203

American Gothic 5, 25, 162, 171-2, 174-5, 177-80, 182-8, 193-7, 198n.7, 198n.11, 198n.17, 198n.20

Amicus 11, 12, 28, 57, 60, 66, 77

Amityville Horror, The 82

And Now the Screaming Starts 80

Andy Williams Show, The 194

Ang, Ien 24, 152-5

Angel 5, 18, 159, 203

Angel Heart 175

anthology drama 13, 22-3, 26-30,

32-43, 47-55, 56n.2, 56n.8, 57-79, $81-6,87-8,90,171$

hosts of 32-3, 34, 35, 38-9, 56n.7, $64,88 \mathrm{n} .4,126$

US anthology drama 13-14, 56n.7, $66,88 \mathrm{n} .4,124-6$

see also individual programmes

Appointment with Fear 32, 39

Archard, Bernard 60

Armchair Mystery Theatre 34, 35, 39

Armchair Theatre 33-4, 37

Ashbrook, Dana 168

Astin, John 127

Asylum 66

audience research 10, 20,91, 151

Austen, Jane 118, 121n.6, 121n.8 
Badalamenti, Angelo 164

Baker, Tom 71

Ball, Nicholas 82

Ballykissangel 97

Barry, Michael 35

Batman 128

Beltran, Henry 39

Bennett, Harve 128

Benshoff, Harry M. 146, 151, 154, 160n.9

Benson, Edward Frederick 29

Beverley Hillbillies, The 130

Beverley Hills 90210174

Bewitched 128, 130, 134, 141-3

Bignell, Jonathan 18

Binchy, Kate 55

Bixby, Bill 130

Black, Lucas 162

Blackwood, Algernon 29, 35, 56n.6

Bloch, Robert 66, 88n.4

Blood From the Mummy's Tomb 80

Blue Velvet 174

Bonham-Carter, Crispin 101

Born, Georgina 21

Botting, Fred 12, 16, 123, 134

Bowen, T.R. 89n.11

Bowles, Peter 55

Brady Bunch, The 194

Brady Bunch Movie, The 194

Bram Stoker's Dracula 88n.3

Branigan, Edward 52

Brett, Jeremy 73, 99

Brideshead Revisited 49

Briggs, Julia 43, 47

Briggs, Susan 134-5

Brimstone 5, 162

British Crime Survey 94

Broadway Television Theatre 125

Bromly, Alan 71-2

Brooks, Peter 144

Brown, Charles Brockden 123

Brunsdon, Charlotte 20, 22, 98

Buck, David 38, 40, 64

Buffy the Vampire Slayer 5, 18, 15960, 198n.6, 202-3

Bundy, Mark W. 203

Burns, Allan 128

Burton, John Nelson 34

Bush, Dick 44
Butler, David 88-9n.11

Buxton, Tim 97

Byers, Frank 164-5

Caldwell, John Thornton 21, 25, 58, 70, 88n.1, 167, 171, 173-5, 176-7, $182,198 \mathrm{n} .10$

see also televisuality

Callander, Michelle 159

Callow, Simon 71

Cant, Colin 87

Capon, Naomi 75

Carnivàle 5, 162

Carr, Terry 96

Carter, Angela 47-8

Carter, Chris 174-6, 177, 180-1, 187, $189,191-3,196-7$

see also Millennium; $X$-Files; The

Cartier, Rudolph 75

Cashmore, Ellis 191-2

'Cask of Amontillado, The' 56n.2

Cassidy, Shaun 174, 193-4, 195-6 see also American Gothic

Cassidy, Ted 127

'Casting the Runes' 54

Castle, Terry 6-7, 13, 172

Caughie, John 36, 66

Chaney Jr., Lon 125-6

Chapman, James 18

Charles, Craig 87

Charmed 141, 160

Cherry, Brigid 10

Chester, Hal E. 56n.13

Chibnall, Steve 11

Children of Green Knowe, The 4, 87

children's Gothic television 86-7, $88 \mathrm{n} .11$

Children's Hour 42

Chiller 4, 51, 87

Christopher Lee's Ghost Story for Christmas 4, 48-9

Cilento, Diane 75

Cinderella 141

cinema $13,14,35,72,141-2$

British 10-12, 18, 23, 28, 57, 60, 64, $66-8,77,78-9,80-1,85-6,172$

early $13,74,172-3$

film noir 2, 167-8

German Expressionism 11, 64-5 
Gothic 1, 8-12, 16-17, 28, 51, 57, $60,64-8,70,77,78-9,80-1,85-6$, 93-4, 97, 101, 105, 121n.4, 126, $128,136,145,146,153,159,161$, $168,172-3,175-6$

heritage 11, 49-51, 100, 121n.8

Hollywood 9-10, 111, 114, 121n.9, $126,128,136,171,173-4,175-6$, 194

horror 10-12, 13-14, 18, 23, 28, 30, $54,57,60,64-8,77,78-81,82$, $85-6,87,125,126,128,136,167-$ $8,174,175-6,202$

social realist 11

terror 12-13, 36

Western 167, 188

woman's film 10, 25n.5, 93-4, 97, 100-1, 105, 120, 121n.4

see also individual films, filmmakers, and production companies

cinematic $9,23,28-9,36,51,58,60$, $64-8,70,81-2,85-6,159,173-7$, 181-3

Clark, Lawrence Gordon 47, 49, 51, 54-5

see also Ghost Story For Christmas

Clayton, Jack 28, 56n.1

Clegg, Tom 88n.10

Clifton House Mystery, The 4, 86

Coates, Paul 11

Coe, Fred 125

Coghill, Ambrose 46

Cole, Gary 162, 194

Collier, Patience 61-3

Collin, Dinah 100

Collin, Reginald 36

Collins, Jim 167

Collins, Norman 29-30

Collins, Wilkie 34, 90, 97-8, 114

competition 33-5, 48-9, 58, 173, 175

Connolly, Joe 128

Connor, Joseph 51

Coogan, Jackie 127

Cooke, Alan 73

Cooke, Lez 29

Coons, Hannibal 144

Coppola, Francis Ford 88n.3

Coronation Street 17
Corsican Brothers, The 172

Count Dracula 4, 59, 72-3

Crawford, Joan 121n.9

Creeber, Glen 21, 26, 166, 167, 170

Creed, Barbara 68, 203

Crisell, Andrew 33, 36-7, 76

Crossroads 22

Culture Show, The 71

Cummings, Bob 130

Curse of Frankenstein 66

Curtis, Dan 13, 146-7, 149-50

see also Dark Shadows

Cushing, Peter 85

Dahl, Roald 74

Dallas 152, 155

Dance, Charles 99

Darkman 174

Dark Shadows (1966-71) 5, 24, 122, $128,134,146-59,158-9$

Dark Shadows (1991) 5, 146

Davenport, Randi 170-1

Davenport-Hines, Richard 12, 16, 123-4, 148-9

David, Joanna 99

Davies, Christopher 52

Davies, Rachel 82

Davis, Anthony 41

Davis, Robert A. 159

Dawidziak, Mark 147-8

Dead of Night 4, 77-8

De Carlo, Yvonne 128

Desperate Housewives 121n.10, 203

Dickens, Charles 47, 55

Dickson-Carr, John 32, 125

Dignam, Mark 40

Doane, Mary Ann 10, 93-4, 105-6

Dodd, Catherine 100

domestic space

American Gothic, in the 123-4, 126

Gothic location, as 1, 3, 10, 11, 14$15,18,24-5,28,38,64-5,70,77-$ $87,90,92-6,98-114,117,120-1$, $131-4,137-45,146,148,151,154$, 155-60, 163-5, 184-6, 188-93, 200

horror of $23,55,72,77-85$

invasion of $140-1,144-5,151,156$, $157-60,163,188,190-3$

prison, as 3, 24, 103-7 
domestic space (cont.)

viewing context, as $7,13-15,17$, 18-21, 22, 24-5, 28, 30-2, 37-9, 42, 47-9, 71, 77, 79, 81, 83, 85, 87-8, 90-2, 94, 95-6, 98, 110-11, $117,120-1,122,124-5,126,129$, $147,151,155,158-9,191-2,200-$ 3

see also family; family, viewers; female spectator; suburbia domestic violence 94, 109-10, 121n.5, 170-1, 196

Donahue, Angela 190

Dors, Diana 85

Dow, Bonnie J. 94

Down, Lesley Anne 72

Doyle, Arthur Conan 74

Dracula (1931) 9

Dracula (1958) 66

Dracula (novel) 9, 59, 66, 71, 72, 125, 149

see also Count Dracula; Mystery and Imagination; Stoker, Bram

Dracula, Prince of Darkness 66-7

Dragonwyck 121n.4

Dr Jekyll and Mr Hyde 149

Dromgoole, Patrick 37, 59, 66, 86

Dr Terrible's House of Horrible 5, 87-8

Dr Who 4, 71, 87, 88n.8

Du Maurier, Daphne 90, 96, 97 see also Rebecca

Dunham, Duwayne 164

Dyall, Valentine 32-3, 35, 56n.4

Dyer, Richard 180, 188

Dynasty 174

\section{Eastenders 97}

Eco, Umberto 19, 91, 92, 121n.3

Eddison, Robert 61-3, 62

Eden, Barbara 130

Edgar Allen Poe Centenary, The 4, 30

Edmundson, Mark 12, 15-16, 161, 197,204

Eisner, Lotte 11

Eligible Bachelor, The 89n.11

Elliott, Denholm 37, 55, 59-61, 60, $68,69,88 \mathrm{n} .3$

Ellis, John 21, 24, 53, 101, 127, 201-2

Ellis, Mundy 116
'Enchanted Cottage, The' 125

Eramo, Steven 198n.7

Eraserhead 174

Evil Dead 174

Exorcist, The 79

experimentation 14, 27, 29, 33-4, 36, 42-3, 58-9, 65-6, 70, 73-6, 78, $177,180-1,203-4$

expressionism 9, 64-6, 153

see also cinema, German

Expressionism

Exton, Clive 55

'Fall of the House of Usher' 56n.2, $123,125,184$

see also Poe, Edgar Allen; Mystery and Imagination

family $1,3,11,15,18-19,23-5,55$, $77-8,79-85,87,92,93-4,98,101$, $102-3,105,107-10,122-4$, 126-30, 134-48, 152, 155-6, 162, 170-1, 184-97, 200, 203

instability of $122,136-45,162$, 170-1, 184-97

viewers $25,37-8,47-9,129-30$, $134-7,171,192-7$

see also domestic space; domestic space, viewing context as; domestic violence; female spectator

fantasy 2, 24, 113, 119, 147, 149, 151-2, 154, 166, 172, 174, 177, $183,198 \mathrm{n} .16$

see also situation comedy, fantastic family sitcom; telefantasy

Farber, Stephen 9

Father Knows Best 80, 129, 137

Faust and Mephistopheles 172

Fawcett Books 96

Feely, Terence $37-8,48,70$

female Gothic 3, 23-4, 25n.5, 50-1, 61, 90-122, 147, 150-1, 154, 191

film 93-4, 105-6, 121n.4

literature 23-4, 90, 92-3, 94-8, 101, $107,109-10,116-20,150$

see also adaptation; and programmes, films, and novels listed individually 
female spectator 10, 22, 24, 90-1, 93-4, 96-8, 110-11, 114-16, 150, $169-70$

see also domestic space, viewing context as; family, viewers

Fenn, Sherilyn 168

Ferguson-Ellis, Kate 92, 95, 96, 101

Feuer, Jane 24, 137

Finch, Jon 81

Fink, Edward J. 175, 177

Firth, Colin 101

Fisher, Terence 11, 66-7

Fitzgerald, Tara 101

Fleenor, Juliann 92

Fleming, Lucy 61, 63

Fletcher, John 10

flow $7,14-15,18,30,58,85,133,167$, 173,203

Flynn-Boyle, Lara 168

Foster, Giles 118-19

Foster, Michelle 52

Fox, Emilia 99

Fox Mystery Theatre 86

Frankenstein (1931) 128

Frankenstein (1973) 5, 160n.7

Frankenstein (novel) 9, 125-6, 149 see also Mystery and Imagination;

Shelley, Mary; Tales of Tomorrow

Freedman, Eric 202-3

Freud, Sigmund 6-7, 15, 80, 130-1, 180, 202, 203

Frid, Jonathan 146, 158-9

Frost, Mark 161-2

Fryer, Peter A. 72

Gallafent, Edward 10

Garcia, Robert T. 127, 128, 142

Gardner, Carl and Wyver, John 26, 59, 63

Garth Merenghi's Darkplace 5, 27, 87

Gaslight 121n.4

Gauntlett, David 20, 91

Gelder, Ken 10

gender roles 122, 127, 129, 138, 141-5, 160

generic hybridity $7-8,24,25 \mathrm{n} .3,71-2$, $109,110,122,126-7,132-4,146$,

$148-9,152,154,159-60,162$,

$167-70,174,202-3$
Genge, N.E. 198n.7, 198n.11

genre study $1-3,5-6,19,22,204-5$

George, Susan 67

Georgian House, The 4, 86

Geraghty, Christine 148

gestural performance $53,60-1,153$

Ghosts 4, 87

Ghosts and Scholars 56n.9

Ghost Story, A 4, 56n.6

Ghost Story For Christmas 4, 13, 22, $27,42,47-55,76$

'Ash Tree, The' 47, 55

'Ice House, The' 47

'Lost Hearts' 51-5, 54

'Signalman, The' 47, 55

'Stalls of Barchester Cathedral, The' 55

'Stigma' 47, 55

'Treasure of Abbot Thomas, The' 55

'Warning to the Curious, A' 51, 55

Ghostwatch 4, 87

Gibson, Alan 79

Gielgud, Val 31-2

Gipps-Kent, Simon 51

Gitlin, Todd 21

glance theory 21, 53

Glen, Iain 102

Goddu, Teresa A. 122-3, 184

Goldbergs, The 129

Gothic literature 2, 3, 8, 9, 16-17, 23, $28,37-8,49,57,119,123,149-50$, $153,159,163$

see also adaptation; American

Gothic; female Gothic, literature

Gothic Phantom 196

Gothic theatre 1, 9, 23, 31-2, 36, 59$64,68,70,153,172$

see also grand guignol theatre; theatricality

Grade, Lew 79

Graham, Charles 59, 66

'Gramercy Ghost, The' 125

grand guignol theatre 28, 57, 62

see also Gothic theatre

Gray, Ann 20, 91

Greene, Sarah 87

Griffiths, Nick 115

Gripsrud, Jostein 106, 163

Gross, Larry 3 
Grunenberg, Christoph 12, 16, 161

Gunning, Tom 13, 172

Gwynne, Fred 128, 132-3, 136

Haddington, Ellie 102

Hagman, Larry 130

Haining, Peter 35, 41-2, 70, 125

Hall, Sam 147-8

Hallowe'en With The Addams Family 160n.1

Halttunen, Karen 124, 188

Hammer 10, 11, 28, 56n.1, 57, 60, 64, 66-8, 78-9, 80, 86, 118-19

Hammer House of Horror 4, 22-3, 77, 78-9, 81-6

'Children of the Full Moon' 82, 85

'Growing Pains' 82

'House That Bled to Death, The' 79, 82-6, 83

'Mark of Satan, The' 79, 82

'Rude Awakening' 79, 82

'Silent Scream, The' 79, 82, 85

'Thirteenth Reunion, The' 79

'Two Faces of Evil, The' 79, 82

'Visitor from the Grave' 79, 82

'Witching Time' 79, 81-2

Hammer House of Mystery and Suspense 78, 86

Hammond, Peter 89n.11

Hanson, Helen 10, 93

Hardy, Robert 119

Hardy Boys Mysteries, The 174, 193-4

Harrison, Taylor 18

Hartley, John 129, 137

Hartman, Mike 164

Haskell, Molly 68

Haunted 4, 77

Haunting of Helen Walker, The 4, 90, 104, 106, 121n.1, 160n.8

Hawkins, Jack 39

Hawthorne, Nathaniel 123

Hayward, Chris 128

Hayward, Susan 114

Heller, Dana 203

Heller, Tamar 96, 97-8

Henessey-Derose, Christopher and

McCarty, Michael 149-50

Henesy, David 147

Henrickson, Lance 162
Hercules 174

heritage drama $8,10,23-4,27,49-51$, $53,98,99-103,109-10,116$

'feel bad' heritage 23, 49, 87

see also cinema, heritage

Hex 5, 198n.6

Higson, Andrew 49-50, 56n.1, 102

Hill, Rosemary 47

Hills, Matt and Williams, Rebecca 203

Hills Have Eyes, The 79

Hinchcliffe, Philip 71

Hoffman, Ernst Theodor Amadeus 131

Hollinger, Karen 10

Holm, Ian 70

Home Service 32, 42

home video 87

Hordern, Michael 42, 44, 45-6

horror 2, 9, 16, 28, 47, 51, 53-5, 57$88,124,132-3,134,136,139,144$, $145,147-8,152,153,159,164$, $167-8,174,190-1,195-7,201-3$

body horror $55,62,67,75-6,84,118-19$ domestic horror 55, 57, 70, 72, 76-86, $154-8,165,171$

sexualised horror 66-8

television horror 3, 7, 13-15, 18, 22-3, 25,25 n. $3,28,29-32,36,51,53-5$, 57-88, 125-6, 162, 176, 190-1, 195-7, 201-3

see also cinema, horror

Hour of Mystery 4, 34-5, 90, 121n.2

House of Dark Shadows 146

House of the Seven Gables, The 123

House That Dripped Blood, The 66

Howells, Coral Ann 92, 96

Hults, Arlo 125

Hush, Hush Sweet Charlotte 9

Huss, Roy 9

Hutchings, Peter 11, 66, 77, 80-1, 82

identification $8,24,77,79,93,95$, $106,111,114-21,140,151-2$, 154-5, 158-9, 191, 200

Idiart, Jeanette and Schulz, Jennifer 123 I Dream of Jeannie 130, 134, 141-3

I Love Lucy 80, 129

impressionism 3, 73-4, 88n.7, 113-14, $163,165-6,177-81,197$ 
impropriety of viewing 30-2, 56n.3, $162,171,192-7,200$

see also family, viewers; female spectator

Innocents, The 28

Inside Daisy Clover 9

intimacy $15,48-9,60,77,94,110$, 114-15, 116-17, 119, 150, 201

I Remember Mama 101

It's Alive 79

Jacobi, Derek 118

Jacobs, Jason 21, 25n.3, 29-30, 59, 163

Jacobs, Seaman 128

James, Ed 128

James, Henry 123

James, Montague Rhodes 13, 26-7, 29, 42-9, 51, 53-6

Jancovich, Mark 10

Jane Eyre 107, 149

Jenkins, Henry 151

Jentsch, Ernst 131, 183

Jewel in the Crown 49

Jivani, Alkarim 194

Johnson, Catherine 18, 22, 177

Johnson, Doris 125

Jones, Carolyn 127

Jones, Freddie 37

Jourdan, Louis 59, 73

Journey to the Unknown 4, 77, 78

Jowett, Lorna 18

Joyrich, Lynne 114-15, 120-1

Justice for Women 94

Kabinett des Doktor Caligari, Das 64

Kahane, Claire 92

Karlen, John 146

Karloff, Boris 88n.4, 125, 126, 128, 136,139

Kaveney, Roz 18

Kemp, Peter 118

Kemp-Welch, Joan 37, 39

Kennedy-Martin, Troy 65-6

Kerr, George F. 37, 39

King, Stephen 13-14, 31

Kingdom, The see Riget

Kingdom Hospital 5, 162

King Lear 32

Kiss of the Vampire 66
Kolchak the Night Stalker 5

Kramer, Wayne 190

Lady's Magazine, The 95

La Rue, Jack 125

Last House on the Left 79

Late Night Horror 4, 22, 25n.11, 27, $58,74-7$

Laurie, John 35, 39, 40

Lavery, David 18

Lawrence, Brian 78-9, 85-6

League of Gentlemen, The 4, 27, 87-8

Leave it to Beaver 80, 128-9, 137

Leaver, Don 79

Ledwon, Lenora 2, 17, 161-3, 167-8, $171,198 \mathrm{n} .1$

Lee, A. Robert 184

Lee, Christopher 48

Lee, Sheryl 162, 169

Le Fanu, Joseph Sheridan 26-7, 61, 90

Lejeune, Anthony 62

Levy, David 128

Lewin, David 119

Lewis, Al 128, 133

Lewis, Matthew G. 28, 57

lifestyle 20, 97-8, 100-1

Light Programme 32, 42, 56n.4

Lights Out 5, 125

Lilith 9

Lincoln, Andrew 115

Lloyd, David 79, 88n.10

Lock, Kate 100

Loring, Lisa 127

Lorre, Peter 125

'Lost Hearts' 54

see also Ghost Story For Christmas

Lovecraft, Howard Phillips 125

Lury, Karen 21, 25n.8, 60-1

Lynch, David 161-2, 164-5, 170, 173-4

McCarthy, Anna 20

MacDermot, Robert 29

McGivern, Cecil 29-30

McGlashan, John 47

MacLachlan, Kyle 162

Madden, Cecil 35

Make Room for Daddy (aka The

Danny Thomas Show) 129

Man in Black, The 56n.4 
Mannoni, Laurent 13, 74, 172, 198n.8

Manoir du diable, Le 172

Marc, David 130, 138

marketing of Gothic television 20-2, $38,43,92,95-8,167$

Martin, Richard 75

Massé, Michelle 93

Masters, William Howell and Johnson, Virginia Eshelman 142

Matheson, Richard 66, 88n.4

Matinee Theater 125

Maude, Joan 56n.2

Mausoleum Club 88n.6

Maxwell, James 60

Meet Me In St. Louis 101

Méliès, Georges 172-3

melodrama 16, 17, 23, 61-2, 67, 115, $120-1,147,148,150,152-5,165$, $167,174,202$

Melrose Place 17

merchandising 97-8, 136, 146

Merck, Mandy 203

Milbank, Alison 92, 95, 96, 121n.6

Mildred Pierce 121n.9

Miles, Robert 92

Millennium 5, 8, 16, 25, 162, 171-2, 174-7, 176, 180-1, 182, 187-93, 195-7, 198n.7, 198n.11

Miller, Jonathan 27, 42-7, 49, 51, 55

Minerva Press 95, 96

Mini-Munsters, The 160n.3

Mirrlees-Black, Catriona 94

model viewer 18-19, 91-12, 110-12, $114,116-21,191,192,200$

Modleski, Tania 10, 92-4, 96, 109-10, 111

Moltke, Alexandra 146

Monk, Claire 50, 100

Monroe, Marilyn 139

montage 3, 44, 64-5, 73-4, 84, 99, $107,113-14,131-2,163,164$, $165-6,168,177-81,184-5,190$, 197

Montgomery, Elizabeth 142

$\operatorname{mood} 3,9$, 36, 41, 52, 65, 125, 163-5

Moondial 4, 87

Moore, Harry 74-6

Morley, David 20, 91
Moseley, Rachel 101, 141-2, 160, 205n.1

Mosher, Bob 128

Muller, Robert 13, 37, 73-4

Mulvey, Laura 120

Munsters, The 5, 16, 24, 122, 126-36, 132-3, 138-41, 145-6, 148

Munsters' Revenge, The 160n.1

Munsters' Scary Little Christmas, The 160n.1

Munsters Today, The 160n.2

Murnau, Friedrich Wilhelm 158

music 16, 41, 51-2, 60, 62, 67, 74, 75, $81-2,84,99,102,107,113,120$, $125,150,154,157,161,164,176$, $178,189,190$

see also sound design

My Favourite Martian 130

My Living Doll 130

My Mother the Car 130

Mysteries of Udolpho, The 119

Mystery and Imagination 4, 13, 22, $27,36-43,48,49,58-70,72,73$, $76,78,86,87,90$

'Canterville Ghost, The' 38

'Casting the Runes' 56n.8

'Dracula' 59-61, 60, 66-70, 69, 86

'Fall of the House of Usher' 38-9, 64-6

'Frankenstein' 70

'Listener, The' 38

'Lost Hearts' 56n.8

'Open Door, The' 38, 39-42, 58, 64

'Room 13' 56n.8

'Sweeney Todd' 63, 64

'Traccate Middoth, The' 56n.8

'Uncle Silas' 61-3, 62

Naremore, James 2

Neane, Gareth 116

Nelson, Robin 166-7

Neve, Suzanne 67-8

New Addams Family, The 160n.2

Newman, Sydney 33-4

Newmar, Julie 130

New Yorker, The 127

Nicholls, Basil E. 30, 32, 43

Night Gallery 5, 126

Nightmare Abbey 121n.6 
Night of Dark Shadows 146

Night of the Demon 28, 54, 56n.13

Night of the Living Dead 79

Night Stalker, The 5, 13, 160n.7

Night Strangler, The 160n.7

Nine Inch Nails 176

Nixon, Nicola 16

Northanger Abbey 4, 90, 118-20, $121 \mathrm{n} .1$

Northanger Abbey (novel) 121n.6

Nosferatu, eine Symphonie des Grauens 64, 158

Nutter, David 175-6, 189, 191

O'Brien, Jim 97

'Oh Whistle and I'll Come to You My Lad' $^{\prime} 2$

Oldman, Gary 88n.3

Oliphant, Margaret 39

Omen, The 79

Omnibus 27, 42

One Step Beyond 5, 126

Ontkean, Michael 169

Oprah Winfrey Show, The 15, 161

oral storytelling 37-8, 47-9, 70

Others, The 5, 162

Out of the Unknown 4, 71-2, 77, 88 n. 6

Owen, Beverly 128

Ozzie and Harriet 80

Paley, Irving 128

Pardoe, Rosemary 56n.9

Parents' Television Council 193

Parker, Lara 149-50, 154

Parkinson, Michael 87

Parks, Lisa 18

parody 16, 87-8, 134, 159, 167

Patrick, Butch 128

Paulson, Sarah 162

Peacock, Thomas Love 121n.6

Peel, John 136

Pepper, Cynthia 144

Pepper's Ghost 198n.9

Petley, Julian 11, 18

phantasmagorias $62,74,172,198 \mathrm{n} .8$

Phantom of the Opera, The 71

Philidor, Paul 172

Photographing a Ghost 172
Pidduck, Julianne 121n.8

Pilkington Report 36-7

Pillai, Alex 115

Pirie, David 11, 23, 28, 57, 114, 115

Pleasance, Donald 39

Poe, Edgar Allen 4, 30, 32, 39, 64-5, $123,125,184$

Point Pleasant 5, 198n.6

police drama $8,167-8,187$

Poltergeist: The Legacy 5, 162

post-feminism 94, 160

postmodernism 16,167

Potter, Jeremy 27

Prawer, Siegbert Sol 12-13, 14, 36, 59, 65

Pride and Prejudice 100-1, 102

Priest, Pat 128

Prior, Allan 43

Probst, Christopher 175-6, 177, 181, 189, 191

Probyn, Elspeth 7, 15, 204

production research 21-2, 91-2

Professionals, The 88n.8

Profiler 5, 162

public service broadcasting 23, 30-2, 36-7, 57, 192, 196

Punter, David 2, 38

Pusey, Fred 63

Quatermass Experiment, The 71

Quick, Diana 104

Radcliffe, Ann 119

radio 30, 32-3, 35-6, 39-41, 42, 56n.4, 56n.6, 56n.9, 57, 124-5

radiophonic 9, 23, 28, 32, 35-6, 39-41, $49,57,125$

Raimi, Sam 174

Ranger, Paul 60, 198n.9

Rankin, Steve 187

Ranshoff, Martin 128

Read, Anthony 79, 81, 88n.8

Rebecca (1940) 10, 97, 121n.4

Rebecca $(1947)$ 4, 97, 121n.2

Rebecca (1954) 4, 97, 121n.2

Rebecca (1979) 4, 90, 97, 99, 104, 106, 108, 113-14, 121n.1

Rebecca (1997) 4, 90, 96-8, 99, 104, 106, 108, 121n.1, 160n.8 
Rebecca (novel) 23, 90, 96-7

Redgrave, Corin 67

Redman, Sarah Jane 190

Richards, Susan 52

Ridley, Emma 82

Riget 7, 25n.3, 198n.12

Robert, Étienne-Gaspard (aka

Robertson) 172

Robertson, Kimmy 169

Rock, Blossom 127

Rogers, Pieter 73

Rohmer, Sax 71

romance, the $8,27,98-9,161,168$

Root, Jane 38

Rosemary's Baby 79

Rosenberg, Max J. 66

Ross, Marion 146

Rowe, Kathleen 142

Russ, Joanna 92, 95-6

Russell, Paddy 75

Sabrina the Teenage Witch 141, 160

Sapphire and Steele 4, 71, 88n.8

Sargent, Dick 142

Sartre, John Paul 139

Sasdy, Peter 79

Saturday Night Stories 56n.6

Saville, Philip 37, 59, 73

Savory, Gerald 74-6, 79

Scharrer, Eric 142

Schlesinger, Katherine 118-19

Schreck, Max 158

Schröeder, Greta 158

science-fiction 71-2, 125-6, 147, 161, 202

Sconce, Jeffrey 133

Scott, Gerry 100

Scott, Kathryn Leigh 154, 160n.5

Screen One 87

Searcy, Nick 195

Sedgwick, Eve 2, 153, 162-3

Seltzer, Mark 193

sensationalism 38, 152-3, 196

serial drama $7,8,12,17,24,26,27$,

$33,68,86,96-7,124,160-99,202$

serial killers $8,16,161,187-92$

Serling, Rod 126

Se7en 175-6

Shakespeare, William 32
Shaw, Don 79

Shelley, Mary 9, 27, 28, 57

see also Frankenstein

Sherlock Holmes 71, 89n.11

Showalter, Elaine 107

showcasing $23,33,36,58,68,70,74-$ $6,172-5$

Shrapnel, John 104

Shubik, Irene 71

Silence of the Lambs 175

Simon, Al 128

Singer, D. 30-1, 32

situation comedy $2,8,19,24,78,80$, $88,122,126-45,157,167-8,188$, 194

fantastic family sitcom 126-7, 130, $134,138,141-3,145$

Six Feet Under 5, 203

Skal, Robert 142

Skeggs, Brian 78-9, 85-6

Slocombe, Christine 87

Smith, George Albert 172

Smitrovich, Bill 190

Snow, Mark 189

soap opera $12,17,22,24,122,134$, $135,146-59,167-70,174,188$

Sobchak, Vivian 147

'Some Words with a Mummy' 56n.2

Sopranos, The 203

sound design 3, 39-41, 44, 65, 78, 111-14, 119, 125, 154, 164, 176, 178-9, 181, 189, 191, 197

see also music

special effects $3,13,22-3,28,44-6$, 57-9, 68-70, 72, 73-4, 175, 182-4, 185-6, 198n.16, 198-9n.17

spectacular mode $13,23,28,36,51$, $54-5,57-77,78,82-6,153,182-3$

Spelling, Aaron 173-4

Spigel, Lynn 18-19, 20, 130, 137-9, 141

Spiral Staircase, The 10

Stacey, Jackie 111

Starr, Mike 190

Star Trek 72

Stevenson, Diane 171

Stevenson, Robert Louis 32

Stoker, Bram 9, 27, 28, 57, 59, 66, $88 \mathrm{n} .3$ 
see also Dracula

Stone, Paul 87

Strange 5

studio set design 40, 63, 64, 64-7, 155-6

subjective perspective $3,20,24,44-6$, $52,102,110-21,150-1,152,155$, $163,165-6,177-81,182,191-2$

Subotsky, Milton 66

suburbia $19,77-8,83,126-7,129-30$, $137-41,143-5,157,190$

suggestive mode $3,9,14,22-3,28,32$, $35-6,39-47,51-5,57,59,68,70$, 181,197

Sunset Beach 17

Supernatural 4, 58, 59, 73-4, 78

'Mr. Nightingale' 73-4

Suspense 5, 125

Sweet, Matthew 71

Tales of Mystery 4, 27, 35, 39

Tales of Tomorrow 125-6

Tansley, Derek 40

teen television 18, 159-60, 167-9, 170, 174, 198n.6, 202

telefantasy $8,17-18,22,71$

see also fantasy

television

of attractions 58

history 1-2, 18

introduction of colour on 22, 25n.11, 63, 74-6

war on $14,30-1,202$

televisuality $25,58,68,70,74,78,87$, 88n.1, 160, 167, 171, 173-84, 190, 191, 194, 197, 198n.10

Texas Chainsaw Massacre 79

textual analysis 19-21, 25n.8, 91-2

textual borrowing 29, 39, 59-68

see also adaptation; cinematic; radiographic; theatricality

theatricality $9,19,23,28-9,53,58$, $59-64,68,70,125,153$

Thomas, Denis 35

Thomas, Howard 34-5

Thriller (1960-62) 5, 56n.7, 88n.4, 126

Thriller (1973-76) 4, 77, 86

Tiballs, Geoff 97

Tiplady, Brittany 189
Todorov, Tzvetan 6, 138, 149

Tom's Midnight Garden 4, 87

Tourneur, Jacques 28, 54, 56n.13

Troubleshooters, The 88n. 8

Tufte, Thomas 20, 91

Turco, Paige 178

Turner, Graeme 8, 19

Turn of the Screw, The (1974) 160n.7

Turn of the Screw, The (1999) 4, 90, 104, 106, 108, 121n.1, 160n.8

'Turn of the Screw, The' (story) 149

TVGuardian 193

Twilight Zone 126

Twin Peaks 5, 16, 17, 24, 56n.12, 16171, 166, 169, 173-5, 193, 195,

198n.2, 198n.3, 198n.6, 202

Two Mrs. Carrolls, The 121n.4

Ultraviolet 4

uncanny $2,3,6-8,10,13,15,16,24$, $42,50,52-3,68,70,71,73-4,106$, $115,126,130-1,133-4,137,153$, $156,158,163,165,169-70,180$, $183,193,200-2,203$

Universal 126, 128, 136

Urban Gothic 5, 87

V-Chip 193, 194

videographic 174, 198n.10

Vidler, Susan 109

viewers' criticisms of Gothic television $35,47,74,76,118$

see also impropriety of viewing

Vitaris, Paula 191, 197

Wadey, Maggie 118

Wagon Train 128

Wakefield, Kimm 176, 181, 182

Waldman, Diane 10, 93, 101

Walker, Amanda 41

Walker, Michael 10, 93

Walking With Dinosaurs 198n.16

Waller, Gregory A. 14-15, 77, 85

Walston, Ray 130

Warner, Marina 116, 172

Warre, Michael 56n.2

Watson, Muse 187

Watts, Naomi 102

Weatherwax, Ken 127 
Weber, Jake 180

Webster, Martyn C. 32

Wednesday Thriller, The 4

Weemote 193

Weiss, Andrea 68

Wheatley, Helen 7, 24, 27, 34, 88n.7, $101,127,198$ n.12

'Whistle and I'll Come to You' 4, 22, 27, 42-7, 51, 52, 55, 56n.10

Whitehead, Colson 193-4

Whitehead, Tony 79

White, Leonard 34, 86

Wieland 123

Wiggins, Maurice 35

Wilcox, Rhonda 18

Williams, Anne 204

Williams, Raymond 202

Williams, Rhys 51

Williams, Tony 80

Wimlett, Trevor 87

Winkler, Harry 144

Wissner, Gary 189

witness 201-2

Wolfit, Donald 34

Wolf Man, The 136

'Woman in White' (1957) 34, 121n.2
Woman in White (1966) 4, 121n.2

Woman in White (1982) 4, 90, 99, 104, 106, 108, 114, 121n.1

Woman in White (1997) 4, 90, 99, 101-2, 104-5, 106-10, 108, $112-$ 13, 114, 115-16, 120, 121n.1, 150, 187

Woman in White (novel) 23, 97-8, 114

Wood, Helen 20, 91

Wood, Robin 79-80

'working through' 8, 24, 93, 101, 127, 201

'worrying at' 24, 93, 101, 109, 127, 151,160

Wunstorf, Peter 175, 181, 189

Wyvern Mystery, The 5, 8, 90, 102-4, 105-8, 115, 116-18, 117, 121n.1, 187

Wyvern Mystery, The (novel) 23-4

Xena: Warrior Princess 174

$X$-Files, The 5, 16, 162, 174, 175, 177, 187,194

Yarborough, Jean 144

Yentob, Alan 192

York, Dick 142 